\title{
Risks and Rewards of Bariatric Surgery in Advanced Chronic Liver Diseases
}

\author{
Yuly P. Mendoza, MD ${ }^{1,2,3, *}$ Chiara Becchetti, $\mathrm{MD}^{1,2, *}$ \\ Annalisa Berzigotti, MD, $\mathrm{PhD}^{1,2, \#}$ \\ ${ }^{1}$ Department of Hepatology, University Clinic for Visceral Surgery and \\ Medicine, Inselspital, Bern University Hospital, University of Bern, \\ Switzerland \\ ${ }^{2}$ Hepatology, Department of Biomedical Research, University of Bern, \\ Switzerland \\ ${ }^{3}$ Graduate School for Health Sciences (GHS), University of Bern, Switzerland \\ ${ }^{4}$ Division of Gastroenterology and Hepatology, Department of \\ Internal Medicine, Mayo Clinic, Rochester, Minnesota \\ Semin Liver Dis 2021;41:448-460.
}

Kymberly D. Watt, MD, PhD ${ }^{4, \#}$

\begin{abstract}
Address for correspondence Annalisa Berzigotti, MD, PhD, Hepatology, University Clinic for Visceral Surgery and Medicine, Bern University Hospital, University of Bern, Switzerland, Murtenstrasse 35, 3008 Berne, Switzerland (e-mail: annalisa.berzigotti@insel.ch).
\end{abstract}

\begin{abstract}
Keywords

- cirrhosis

- nonalcoholic fatty liver disease

- sarcopenia

- malnutrition

- alcohol use disorders

The burden of obesity and metabolic syndrome has determined a sharp increase in bariatric surgery (BS) procedures, which lead to marked weight loss, improved metabolic syndrome, reduced cardiovascular risk, and even improvement in nonalcoholic steatohepatitis (NASH). Despite these promising results, BS in patients with chronic liver disease can rarely lead to worsening of liver function, progression to cirrhosis and its complications, and even liver transplantation. On the other hand, since obesity in patients with cirrhosis is a major cofactor for progression to a decompensated stage of the disease and a risk factor for hepatocellular carcinoma, BS has been used to achieve weight loss in this population. In this review, we critically analyze the existing data on outcomes of BS in patients with cirrhosis and the possible mechanisms leading to fibrosis progression and worsening liver function in patients undergoing BS. Finally, we propose a set of measures that could be taken to improve the multidisciplinary management of liver disease in patients undergoing BS, including early recognition of malnutrition and alcohol misuse.
\end{abstract}

\section{Rationale for Bariatric Surgery in Patients with Cirrhosis}

The obesity epidemic has grown dramatically, becoming a public health concern. ${ }^{1}$ Since sustained weight loss with lifestyle modifications alone remains difficult for many individuals with severe obesity, bariatric surgery (BS) procedures (also recently termed "metabolic surgery") have become increasingly common. ${ }^{2}$ The liver-related benefits resulting from BS are mostly related to the marked weight loss following these procedures. Additionally, glucagon-like

* Yuly P. Mendoza and Chiara Becchetti equally contributed and share the first authorship.

\# Kymberly D. Watt and Annalisa Berzigotti equally contributed and share the senior authorship.

published online

July 9, 2021
DOI https://doi.org/ 10.1055/s-0041-1731705. ISSN 0272-8087. peptide-1 (GLP-1) levels, which increase satiation, improve insulin sensitivity, and alter the modulation of bile acid signaling, increase after BS, and this has been linked to a positive shift in the gut-liver axis mediated by Farnesoid $X$ receptor. ${ }^{3,4}$ Long-term benefits of BS include long-term weight loss, remission of metabolic risk factors such as diabetes, dyslipidemia, and hypertension, ${ }^{5}$ and improved long-term mortality. ${ }^{6}$ In patients with cirrhosis and obesity, weight loss targeting $10 \%$ of body weight is a major therapeutic goal, as it associates with NASH histologic improvement and, since obesity per se associates with higher risk of decompensation, hepatocellular carcinoma, portal vein thrombosis, and acute on chronic liver failure. ${ }^{7}$

Patients with obesity who meet the criteria for BS, namely those with body mass index (BMI) $>40 \mathrm{~kg} / \mathrm{m}^{2}$ or $\geq 35 \mathrm{~kg} / \mathrm{m}^{2}$

\footnotetext{
(c) 2021. The Author(s).

This is an open access article published by Thieme under the terms of the Creative Commons Attribution-NonDerivative-NonCommercial-License, permitting copying and reproduction so long as the original work is given appropriate credit. Contents may not be used for commercial purposes, or adapted, remixed, transformed or built upon. (https://creativecommons.org/ licenses/by-nc-nd/4.0/)

Thieme Medical Publishers, Inc., 333 Seventh Avenue, 18th Floor, New York, NY 10001, USA
} 
and at least one or more obesity-related co-morbidities frequently have features of nonalcoholic fatty liver disease (NAFLD) or nonalcoholic steatohepatitis (NASH)/metabolic liver disease (MAFLD) ${ }^{8,9}$ Indeed, BS has been proved to improve long-term survival and death from cardiovascular disease (CVD) and malignancy, the two most frequent causes of death in patients with NASH. ${ }^{10}$ Several prospective and retrospective cohort studies in NASH patients have shown that sustained weight loss associates with reductions in steatosis, inflammation, and fibrosis, already evident 1 year after $\mathrm{BS}^{11-14} \mathrm{~A}$ recent meta-analysis from observational studies, which did not include patients with cirrhosis, showed that $66 \%$ of patients achieved steatosis improvement, 50 and $76 \%$ of patients improve the ballooning and inflammation, respectively, and $40 \%$ of patients experienced improved fibrosis. Interestingly, however, $12 \%$ of patients showed de novo NAFLD and worsening of fibrosis. ${ }^{15}$

\section{Progression of Liver Fibrosis after BS and Feasibility and Safety of BS in Patients with Cirrhosis}

Despite the promising results of BS in NAFLD and its increased use, there has been increased awareness of BS complications. A systematic review showed that worsening liver disease belongs to the spectrum of complications of $\mathrm{BS}^{16}$ and recent case series have reported the onset of cirrhosis complicated by liver failure leading to liver transplantation (LT). ${ }^{17-20}$ To date, one study specifically looked at the efficacy and safety of BS in patients with compensated cirrhosis with the majority of their patients having NASH/MAFLD. ${ }^{21}$ This sleeve gastrectomy study showed excellent weight loss, and metabolic improvement with no significant complications despite some patients having mild portal hypertension on endoscopy. The American Gastroenterological Association (AGA) recently published a clinical practice guideline for BS in cirrhosis, suggesting that BS can be considered in selected patients with compensated cirrhosis but should only be performed after careful evaluation and management of extrahepatic comorbidities, and after assessing portal hypertension. ${ }^{22}$

Indeed, in-hospital mortality rate after BS is higher in patients with compensated cirrhosis versus patients without cirrhosis ( 0.9 vs. $0.3 \%$ ), and markedly increased in patients with decompensated cirrhosis (16.3\%). ${ }^{23}$ In a recent U.S. nationwide population-based cohort, among 1,679,828 patients undergoing BS, 9,802 patients had cirrhosis. ${ }^{24}$ Mortality was $1.81 \%$ in patients with cirrhosis and $0.17 \%$ in those without cirrhosis. However, the authors reported a change in the type of BS over the study period, shifting from mixed procedures (RYGB and biliopancreatic diversion-BDP) to restrictive procedures (SG, vertical banded gastroplasty, and adjustable gastric banding-AGB) resulting in a lower in-hospital complications and decreased mortality. ${ }^{24}$

The type of surgery is one of the criteria that should be considered in balancing the risks and benefits of BS in cirrhosis. A systematic review of the outcome of 122 patients with compensated cirrhosis (96.5\% Child-Pugh A, and 3.4\%
Child-Pugh B) undergoing BS showed that mortality related to BS was only observed in BPD and RYGB group at 20 and $3.9 \%$, respectively. ${ }^{25}$ No mortality was observed with SG and AGB. Overall, nine patients (7.3\%) decompensated after surgery, but all the decompensating episodes in SG patients were self-limited, and none resulted in mortality. ${ }^{25}$ Longterm data on the outcome of these patients is not available, and specifically, it is not known to what extent cirrhosis can regress in this situation. Long-term follow-up of a small cohort of compensated cirrhotic patients with sleeve gastrectomy over 8 to 10 years did show sustained weight loss, stable liver function with only one patient developing hepatic encephalopathy (with coexisting mania complicating the diagnosis) 3 years after BS. No progression of disease or liver dysfunction was noted over the 10-year follow-up. ${ }^{26}$

Based on the published case series and on data regarding elective extrahepatic surgery, ${ }^{27}$ severe portal hypertension should be seen as a contraindication to BS. Two small series showed that BS might be safe in cirrhosis patients and mild portal hypertension, but one study reported a retrospective experience, and both were limited to a single tertiary center. ${ }^{21,28}$ Further large-scale studies are warranted to evaluate the safety and outcomes of BS in patients with cirrhosis and portal hypertension, and the current AGA clinical practice guidelines recommend avoiding BS in this situation. ${ }^{22}$

Based on the existing data, patients with compensated cirrhosis (Child-Pugh class A) without severe portal hypertension might be eligible for BS, and SG might be more suitable in this setting. The decision should be taken on a case-by-case basis, after having carefully assessed portal hypertension. ${ }^{10,22}$ BS candidate patients with known cirrhosis or signs of bridging fibrosis would benefit from measurement of the hepatic venous pressure gradient (HVPG), which is the gold-standard method to evaluate portal hypertension in patients with chronic liver disease. ${ }^{29}$ If this is not available, upper gastrointestinal endoscopy to identify/rule-out varices should be utilized.

Historically BS procedures with a marked malabsorptive component, such as jejunoileal bypass (JIB) or BPD (Scopinaro procedure) were proven to cause severe life-threatening complications including acute liver failure in up to $10 \%$ of patient, and were therefore subsequently abandoned. ${ }^{30,31} \mathrm{In}$ a nationwide Belgian survey in 2010, 10 patients (nine of them with BPD and one JIB) were listed for LT due to severe liver failure after BS, with a median time of 5 years after the weight-loss surgical procedure. The authors concluded that, although rare, the BPD operation carries a potential risk of life-threatening liver failure. ${ }^{32}$

More recently, Addeo and coworkers published a review that included 14 studies, reporting an overall 36 patients listed for LT after BS (mainly JIB and BPD). Liver failure developed after a median of 20 months post-BS and was significantly shorter after BPD. Four patients died while on the waiting list for LT, and four additional patients died after LT; 21 patients had their BS procedure reversed, mostly simultaneously with LT. Explant histology documented advanced fibrosis/cirrhosis, as well as necrosis and massive steatosis. Additionally, biopsy-proven steatosis recurrence 
after LT was reported in six patients, of whom four did not undergo BS reversal. ${ }^{19}$

Because of this potentially fatal complication, malabsorptive procedures have been progressively abandoned in favor of restrictive procedures, more often achievable through laparoscopic or mini-invasive approach (i.e., laparoscopic adjustable gastric banding and SG), ${ }^{33}$ or procedures combining malabsorption and restriction (i.e., RYGB). New evidence shows that liver damage progression can occur even after these restrictive or partially restrictive procedures. ${ }^{20,34}$ In this respect, a secondary analysis of a trial comparing the effects of SG versus RYGB on liver function in bariatric patients with NAFLD has shown that at 1 month after BS, albumin value and INR were significantly more frequently impaired in the RYGB group than in the SG group. Conversely, at 12 months, the improvement in transaminases and gamma-glutamyl transpeptidase (GGT) was more evident for SG. The authors concluded that NAFLD patients undergoing RYGB may be more prone to develop early transient deterioration of liver function than after SG. ${ }^{35}$

In two recent studies, liver decompensation after BS was described as an increasingly observed event, associated with two key factors: malnutrition and alcohol use disorders. ${ }^{20,34}$ In the Swiss series, 3 out of 17 post-BS decompensated patients underwent LT, whereas two were still on the waiting list at the end of follow-up. ${ }^{20}$ In the Belgian study, the authors compared patients listed for LT for alcoholic liver disease with or without previous BS. They found that BS patients were younger, with more severe decompensation and a significantly shorter timeframe between diagnosis, listing, and LT. Additionally, they showed comparable survival and post-LT complication rates between patients with and without BS. ${ }^{34}$

\section{Bariatric Surgery and Liver Transplantation}

Considering that NASH/MAFLD is becoming the most emerging indication for LT among adults, according to the European Liver Transplant Registry (ELTR) and the United Network for Organ Sharing (UNOS) databases, ${ }^{36,37}$ it can be expected that an increasing prevalence of future LT candidates may have had BS prior to LT. BMI $\geq 40 \mathrm{~kg} / \mathrm{m}^{2}$ is still considered a surgical contraindication to LT in many transplant programs, thus, BS may allow access to the LT waiting list to morbidly obese patients with end-stage liver disease at more transplant centers.

Several studies addressed the clinical impact of prior BS on post-LT outcomes. Serrano et al reported a similar length of hospital stay and ICU stay after LT (5.3 vs. 4.1 days, $p=0.16$ ), a similar 30 -day complication rate (76 vs. $85 \%$, $p=0.43$ ), and similar 1 - and 3-year survival in patients with or without prior BS. On the other hand, data regarding survival on the waiting list for LT after BS are controversial. Idriss and coworkers documented a higher rate of dropout and death on the waiting list among patients with prior BS ( 33.3 vs. $10.1 \% ; p=0.002$ ), and lower LT rate ( 48.9 vs. $65.2 \%$; $p=0.03)$. However, in the presented cohort the most common BS procedure was RYGB (63\%). ${ }^{38}$
The feasibility of pre-LT SG was studied in well-compensated LT candidates with median MELD score of 12 , not achieving an adequate weight reduction through a medically supervised program. The authors did not observe deaths or liver-related morbidity, and after 6 months, 28 out 32 (88\%) candidates were deemed eligible for $\mathrm{LT}^{39}$

Recently, LT and simultaneous BS procedures such as SG have been demonstrated feasible, with an acceptable rate of perioperative complications, ${ }^{40}$ and good long-term outcome, showing a reduction of the post-LT rate of obesity and metabolic complications. In a cohort of 29 patients (average MELD at LT of $32.0 \pm 9.5$ points) that underwent simultaneous LT and SG the authors reported two liver-related deaths (recurrent hepatocellular carcinoma and cholestatic allograft failure), one re-LT (early hepatic artery thrombosis), and three re-operations due to bleeding, not related to the SG. Gastric leak, severe gastroesophageal reflux, and persistent renal failure are also reported, with more likely relation to the SG component of the operation. However, when the follow-up data are compared with the cohort of patients with medically complicated obesity, who achieved weight loss target through lifestyle modification and underwent LT alone, no difference in term of survival was found. ${ }^{41}$

The optimal timing to perform BS in LT candidates still remains a controversial topic. An approach to BS in LT candidates should be individualized. BS might be considered prior to LT in situations where the weight loss may provide an advantage of improving eligibility for LT. As discussed, this approach may be feasible in compensated patients (Child A or $B 7, M E L D<15$ ) listed with MELD-exceptions, without signs of severe portal hypertension, ${ }^{42,43}$ but should not be proposed to patients with more severe liver failure or portal hypertension.

The published data on the simultaneous BS + LT show this technically feasible, but complications in the postoperative phase are possible (stapleline leak, N/V, and GERD). ${ }^{41}$ Detailed discussions on the risk and benefit of the combined procedure are advised. In addition, a multidisciplinary team with specific experience in BS is crucial in achieving good outcomes.

BS after LT aims to treat morbid obesity in efforts to reduce the risk of metabolic complications after LT and improving long-term outcomes. Reported series described an interval from LT to BS ranging between 27 and 70 months, ${ }^{44,45}$ and thus the safety and effectiveness of BS within the first 2 years of transplant are unknown. Special care with respect to immunosuppression should be taken on planning BS after LT, with restrictive procedures having less effect on absorption than malabsorptive procedures (which may require more careful immunosuppression follow-up). Taken together, however, published data suggest that BS does not significantly alter immunosuppression levels and doses. ${ }^{45}$ As with any surgical procedure, patients on mammalian target of rapamycin inhibitors (mTOR inhibitors) would need to be switched to other immunosuppression regimens, ${ }^{46}$ due to the high risk of wound complications in patients on these drugs.

The overarching conclusion from this review shows, that evidence suggesting a proportion of BS patients, especially 
those receiving malabsorptive $\mathrm{BS}$, may experience a rapid deterioration of liver function such as to require LT. New findings seem to highlight that severe malnutrition and alcohol use disorder likely have a role in this progression. Therefore, close follow-up of patients with underlying chronic liver disease after BS is required to early counteract or reverse this progression and avoid, when possible, the need for LT.

\section{Pathophysiological Mechanisms Potentially Driving Fibrosis Progression and Clinical Decompensation after BS}

As mentioned, malnutrition is associated with severe episodes of decompensation of liver disease after BS. ${ }^{20}$ The histological lesions of lethal cases after initially used BS procedures, as JIB, were severe steatohepatitis ${ }^{47,48}$ (-Fig. 1). As for the radiological findings, in the case series of patients presenting with liver decompensation, ${ }^{20}$ among 17 patients, four showed significant hepatomegaly in computerized tomography at time of decompensation (-Fig. 1, panel E). All of these patients had no prior known liver disease, underwent RYGB, and had a severe presentation of decompensation, which required nutritional support.

The mechanism leading to decompensation of liver disease in the context of malnutrition or rapid weight loss after BS remains poorly understood (-Fig. 2 ).

\section{Rapid Weight Loss and Malnutrition}

Malnutrition and malabsorption are the two main pathophysiologic factors driving liver damage in patients undergoing BS. It has been postulated that protein and amino acid malnutrition can rise or prolong lipid accumulation in the liver, leading to exacerbation of preexisting steatosis. ${ }^{32}$ The rapid and excessive weight loss after BS produces fast and massive destruction of adipose tissue in visceral and peripheral deposits, leading to the release of free fatty acids (FFAs) into plasma, reaching the liver through the portal vein. $^{49,50}$ This increases $\beta$-oxidation, de novo lipogenesis, inflammation, and necrosis in the hepatocytes. ${ }^{50}$ Alternatively, it has also been hypothesized that after BS with a malabsorptive component, bacterial overgrowth of the excluded small intestine and alterations in gut permeability would lead to spill-over of endotoxins, reaching the liver and triggering inflammation-related liver damage. ${ }^{32}$ However, experimental data supporting this hypothesis are currently lacking.

Malabsorption of nutrients, vitamins, and trace metals is a common postoperative complication in BS patients. A recent study showed that the presence of malnutrition as well as sarcopenia may contribute to worse outcomes in patients with a previous BS on the LT waiting list. ${ }^{38}$ Sarcopenia in obese patients is thought to be partially mediated by adipose tissue-associated inflammation. ${ }^{51}$ The persistence of excess adipose tissue after BS might perpetuate this mechanism, which can be further aggravated by lack of mechanisms promoting muscle anabolism (e.g., insufficient protein intake; excess of muscle catabolism during quick weight loss; lack of exercising due to deconditioning). New insights have highlighted that sarcopenia is significantly associated with NASH and fibrosis, even in the early stage of the liver disease. ${ }^{52}$ These findings have underlined the concept that muscle quality matters over quantity and prompted the hypothesis that sarcopenia may play an etiological role in NASH. Insulin resistance (IR) and increased inflammation play a key role in the development of both conditions. Furthermore, myokines secreted by skeletal muscle (IL-6 and Irisin) are recognized to be involved in regulation of some metabolic variables, like the control of weight gain and the IR. ${ }^{53}$

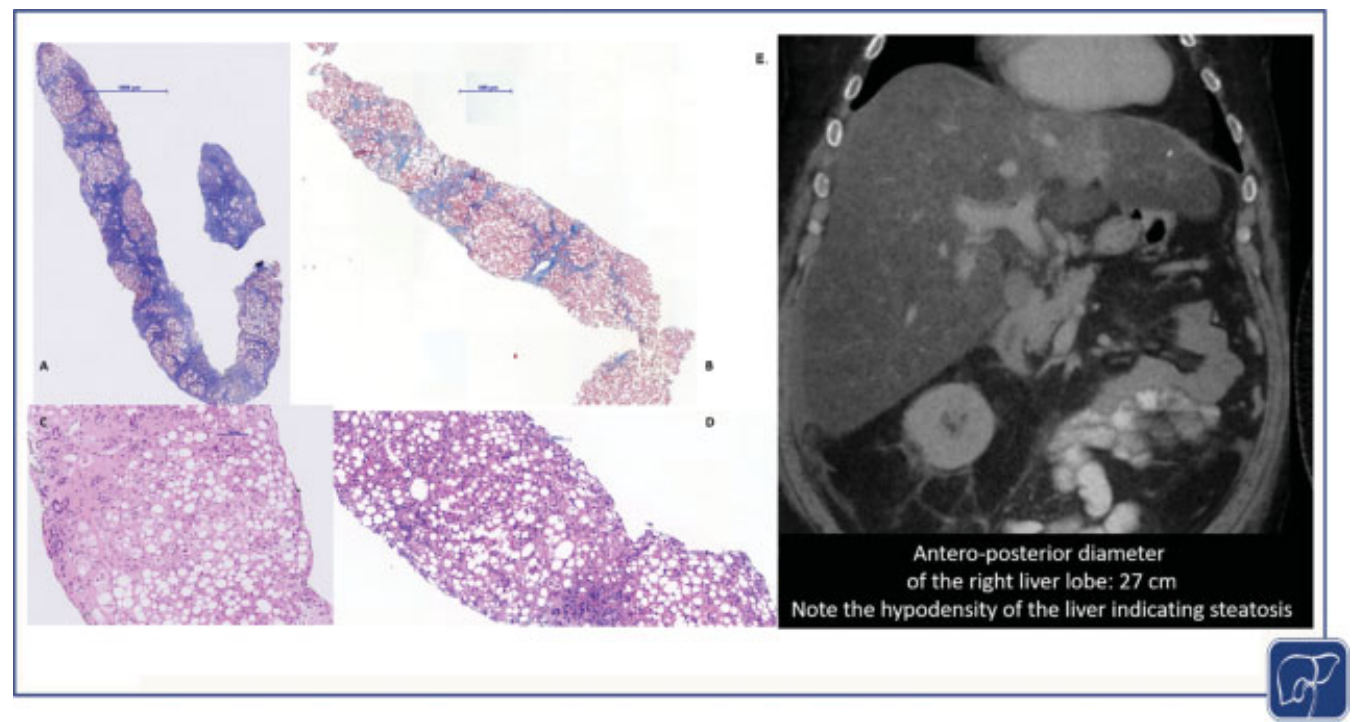

Fig. 1 Histological and radiological findings in patients with severe liver complications after RYGB. (A, B) Woman, 31 years of age, with intraoperative liver biopsy without fibrosis, the figure shows the second biopsy obtained during the liver decompensation ( 3 year after surgery). It showed cirrhosis associated with severe steatosis, steatohepatitis, and cirrhosis. (C, D) Woman, 45 years of age, biopsy 4 years after BS showing high-grade steatosis and steatohepatitis with fibrous septa and remodeling typical of cirrhosis. (E) The CT showed large hepatomegaly, severe steatosis, and signs of cirrhosis, and portal hypertension. 


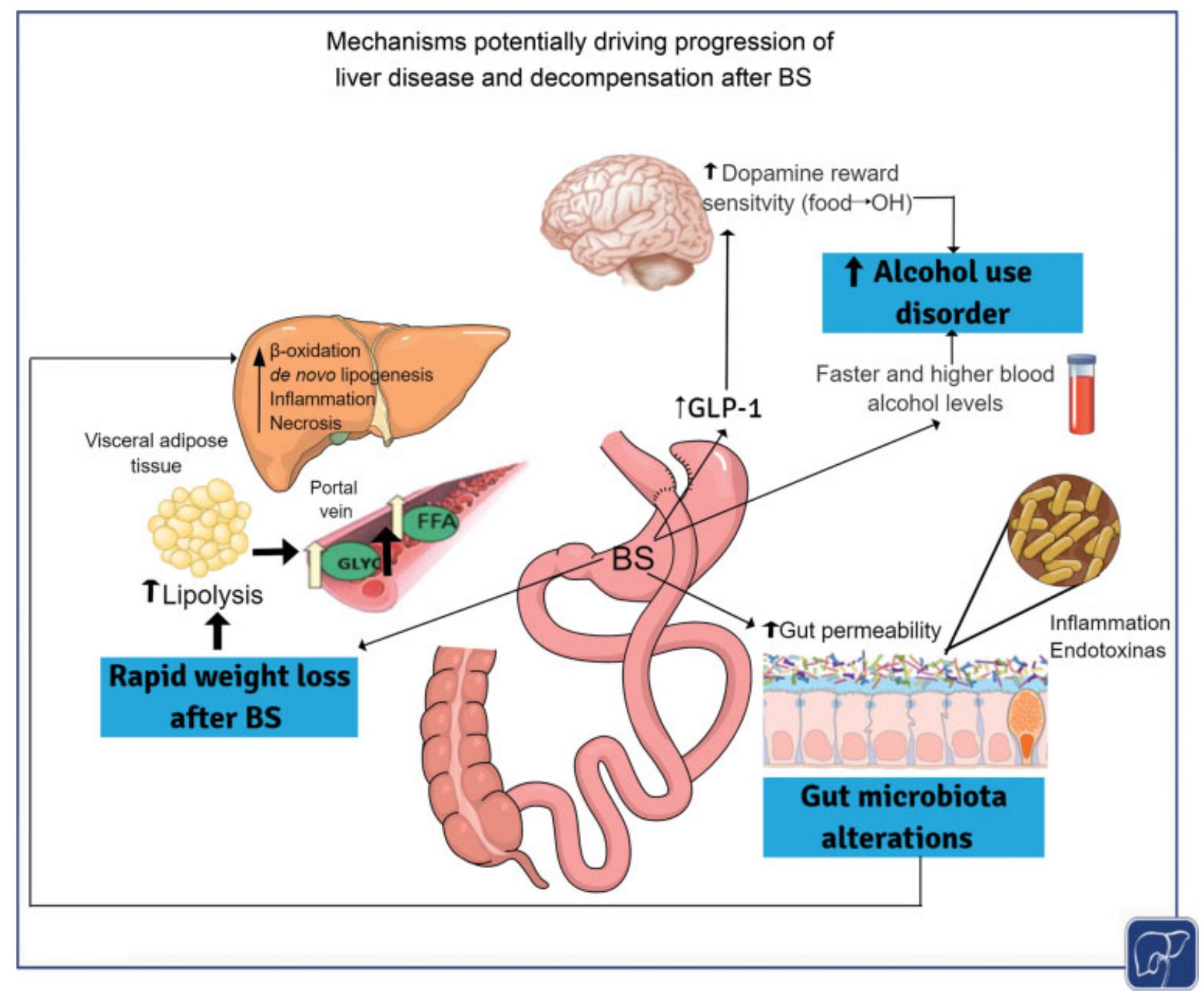

Fig. 2 Possible pathophysiological mechanisms of liver complications after BS. BS, bariatric surgery.

\section{Alcohol}

Experimental and clinical evidence suggests that BS increases the risk of developing an alcohol use disorder, ${ }^{54-56}$ with rates ranging from 8.5 to $21 \%,{ }^{57,58}$ including cases of de novo alcohol use disorder. ${ }^{20,59}$ As compared with other surgical techniques, the prevalence of alcohol use disorder is higher after RYGB. ${ }^{54,55,58,59}$ The hypothesized mechanism includes "addiction transfer" in which patients replace one addiction (food) for a new addiction (alcohol) after BS. ${ }^{60}$ It has been hypothesized that neuro-hormonal shifts after BS determine changes in reward processing and dopamine signaling, ${ }^{61}$ and preliminary evidence from rats and imaging studies in humans has suggested modifications in brain dopamine functions following BS. ${ }^{62-64}$ In addition, hormones as ghrelin and GLP-1 change after BS and play a role in the gut-liver-brain axis modulating the dopamine reward system. BS would then be followed by an increased sensitivity to rewards alternatives to food, such as alcohol. $^{61,65}$

Alterations in pharmacokinetics following RYGB and SG also likely play a role. BS accelerates alcohol absorp- tion and warrants longer time to eliminate alcohol, inducing a lower alcohol tolerance and leading to a faster intoxication with less alcohol. ${ }^{66}$ It is well established that the more rapid a substance reaches the brain, the higher the risk of its addictiveness. ${ }^{67}$ Therefore, increased sensitivity to alcohol after BS possibly influences a higher risk of alcohol use disorder following BS. ${ }^{65}$ In a recent population-based cohort of 8,966 patients followed up for 7 years, the risk of alcohol dependence after RYGB was almost double the risk compared with SG (incidence rate ratio 1.8 [95\% CI 1.1-2.8], for RYBG versus control, $p=0.012$, and 0.6 [95\% CI 0.3-1.1] for SG versus control, $p=0.073)^{6}$

Finally, critical social factors such as depression play a role on increased alcohol consumption. The increased reward sensitivity combined with unsolved presurgery factors (e.g., impulsiveness, binge-behavior traits) and post-surgery conditions (e.g., depression, psychosocial factors) might enhance the risk of new onset of alcohol use disorder. ${ }^{61}$ These problems typically tend to develop approximately 1 to 2 years after BS. ${ }^{54,58}$ 


\section{Pre- and Postoperative Evaluation and Management of Patients with Chronic Liver Disease and Cirrhosis}

\section{Assessment of the Presence and Severity of Liver Disease}

The presence and severity of liver disease should be carefully assessed prior to BS. Alcohol consumption and chronic viral hepatitis should also be evaluated. NAFLD can be screened by using continuous attenuation parameters (CAP score), ultrasound (or other imaging methods), and fibrosis assessments by simple noninvasive tests such as NAFLD Fibrosis Score (NFS) or fibrosis-4 index (FIB-4), alone or combined with liver stiffness measurement (LSM) by transient elastography-TE (using the XL probe), ${ }^{68}$ which can help rule out severe fibrosis and cirrhosis. ${ }^{10,69,70}$ Despite data in the specific setting of patients candidate to BS are scarce, in the NASH population, an LSM cut-off of less than $8 \mathrm{kPa}$ could reliably exclude advanced fibrosis and cirrhosis with a 94 to $100 \%$ negative predictive value ${ }^{70,71}$; on the other hand, values above 12 to $15 \mathrm{kPa}$ have a high positive predictive value (ranging from 80 to $90 \%$ ) to detect advanced fibrosis or cirrhosis. If advanced fibrosis or cirrhosis is diagnosed, further diagnostic tests to address the presence of portal hypertension are required to stratify the risk of surgical complications and mortality. If BS is still an option, restrictive procedures, such as the sleeve gastrectomy, should be preferred (-Fig. 3 ).

In cases where transient elastography does not provide valid results, measurement of liver stiffness using twodimensional share wave elastography (2D-SWE) or magnetic resonance elastography (MRE) can be considered. MRE has a higher success rate and accuracy than ultrasound-based technologies ${ }^{70}$ but is limited by cost and center specific lack of available devices to assess morbidly obese patients.

In case noninvasive tests suggest cirrhosis (the choice of the cut-off will depend on center-specific strategies maximizing the sensitivity or the specificity of the tests), liver biopsy may be indicated to rule-in/rule-out this diagnosis. The transjugular route might be preferable to minimize risk in obese patients. ${ }^{72}$ In addition, this route allows the simultaneous measurement of the HVPG, allowing portal

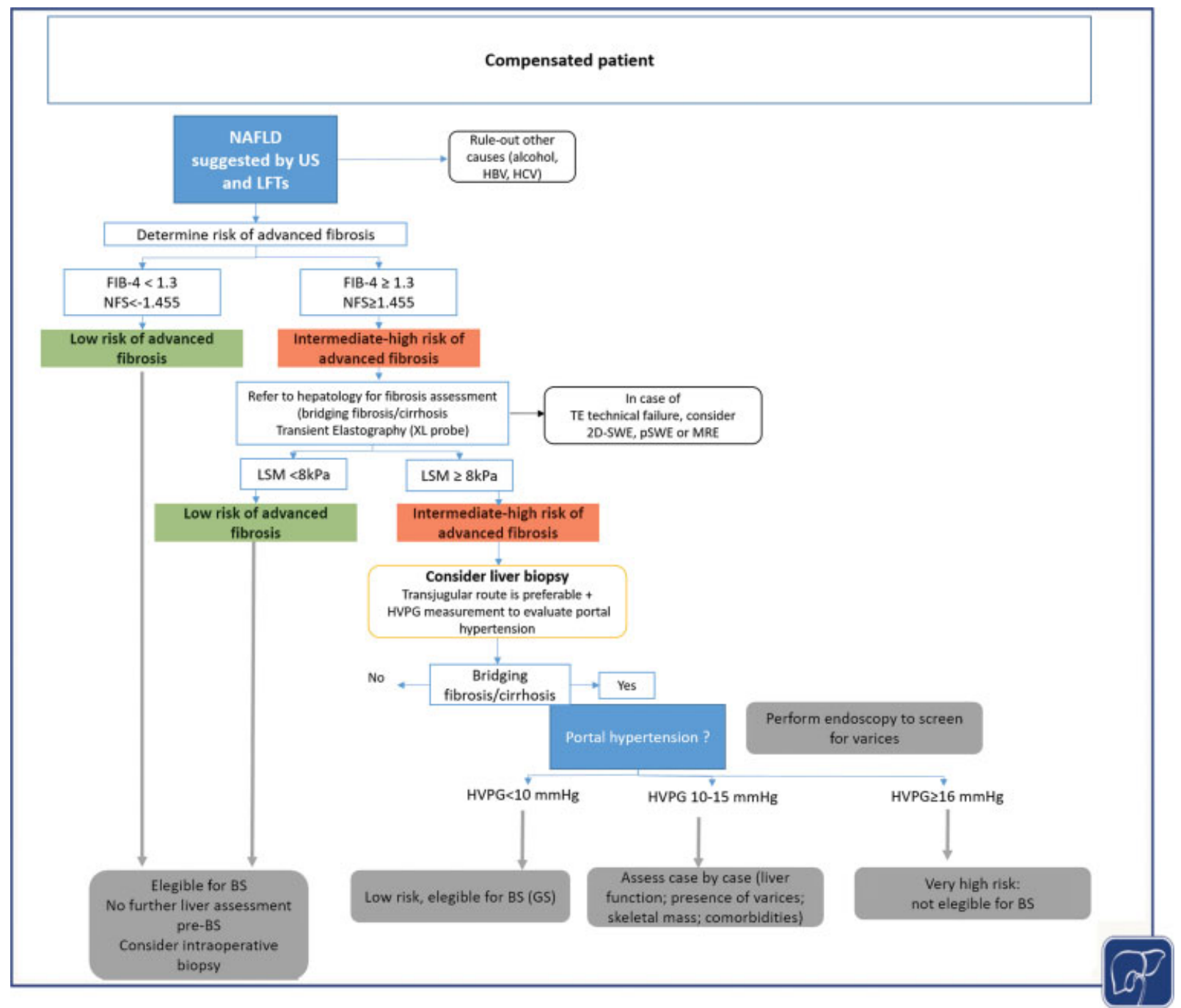

Fig. 3 Suggested algorithm for the assessment of the presence and severity of liver disease preoperatively of BS (Adapted from Newsome et al ${ }^{9}$ and Castera et $\mathrm{al}^{70}$ ). 
hypertension assessment, which is a crucial factor while assessing surgical risks. A recent study assessed the prognostic role of HVPG in cirrhotic patients undergoing elective extrahepatic surgery. The authors showed that HVPG $>16$ $\mathrm{mm} \mathrm{Hg}$ is associated with higher 1-year mortality and an HVPG $>20 \mathrm{~mm} \mathrm{Hg}$ identified a subset of patients at very high risk of death (44\%). ${ }^{27}$ Whether this also applies specifically to BS requires further study, but until those studies are performed it is reasonable to follow this guidance.

The presence of splenomegaly associated with thrombocytopenia, and the presence of gastroesophageal varices on endoscopy can be considered surrogate signs of clinically significant portal hypertension. Other proposed parameters useful for preoperative risk stratification include the ChildPugh classification, MELD score, the ASA class, the Mayo postoperative mortality risk, and the VOCAL-Penn model (www.vocalpennscore.com). ${ }^{74}$ These tools provide additional information to clinicians and the surgical team to guide the decision of whether to perform the intervention. ${ }^{74}$ However, specific calibration of these models for BS is not available.

Presence of ascites indicates a decompensated stage of cirrhosis, with very high risk of further decompensation and mortality. In patients with decompensated cirrhosis and morbid obesity, LT should be considered. ${ }^{74}$

Assessment of liver disease after BS requires routine liver tests including bilirubin, transaminases, GGT, INR, and albumin. Patients with an intraoperative diagnosis of NASH and significant/bridging fibrosis or cirrhosis should be referred to liver specialists for further evaluation. Patients with known cirrhosis should undergo hepatocellular carcinoma screening and be closely monitored to prevent episodes of decompensation.

\section{Alcohol Assessment}

The incidence of alcohol consumption increases over the postoperative period of $\mathrm{BS}^{20,54} \mathrm{~A}$ recent prospective study based on 5,724 patients observed an increase in alcohol use disorder incidence in patients undergoing SG and RYGB during the first 2year of follow-up. ${ }^{57}$ Female gender and RYGB are risk factors for alcohol-related cirrhosis after BS. ${ }^{58}$ However, another study described a similar risk for alcohol use disorder patients undergoing SG and RYGB. ${ }^{57}$ Preoperatively, patients should be educated about possible alcohol metabolism changes that may make them more susceptible to new or worsening alcohol abuse after BS. ${ }^{75,76}$ Moreover, the guidelines recommend careful preoperative screening for alcohol misuse. ${ }^{77}$

Based on the British Society of Gastroenterology and British Association for the Study of Liver disease, the first step would be to identify harmful drinking patterns (greater than 50 units per week for men and 35 units per week for women). For these high-risk groups, the next critical step is to assess liver fibrosis and consider referral to the alcohol service. ${ }^{69}$ For patients drinking less than this, initial screening with the brief version of Alcohol Use Disorders Identification Test (AUDIT)-C score is recommended (-Supplementary Table S1, available in the online version only); a patient scoring $\geq 5$ should proceed to the full 10 item AUDIT questionnaire. A full AUDIT score $\geq 8$ warrants brief alcohol intervention and follow-up, but a full AUDIT score $>19$ is considered a higher-risk group and referral to the alcohol service is recommended. ${ }^{78,79}$

When alcohol use disorder is diagnosed prior to surgery, abstinence should be recommended and monitored, and patients should undergo a formal multidisciplinary evaluation before performing BS. ${ }^{77}$ Postoperatively, clinicians and mental health professionals following-up patients who underwent BS should actively ask about alcohol consumption and apply the AUDIT-C screening. ${ }^{80}$ Regular control with routine liver tests such as GGT may increase the detection of alcohol consumption. If there is doubt regarding alcohol misuse and if an increase in liver tests might be attributable to alcohol consumption, this could be screened either with serum carbohydrate-deficient transferrin, with phosphatidyl ethanol, or with urinary ethyl glucuronide. ${ }^{81}$

\section{Mental Health Assessment and Social Factors}

Psychosocial factors affect long-term outcomes of BS. They can affect adherence to the postoperative recommendations, weight loss outcomes, and comorbidity improvement. ${ }^{82}$ Therefore, a pre-BS psychological assessment is required in the majority of the BS programs. Domains for presurgical psychosocial evaluation that need to be explored include weight history, eating disorder symptoms (i.e., binge eating or night eat disorders), social and family support, cognitive function, and structured psychiatric diseases, mainly depression and anxiety. ${ }^{82} \mathrm{~A}$ study conducted by the Longitudinal Assessment of Bariatric Surgery Research Consortium on 199 patients followed up for 3 years has shown a decrease in psychiatric disorders after BS. However, the presence of post-BS eating disorders was associated with suboptimal weight loss. ${ }^{83}$ The same population followed-up for 7 years showed that mood and anxiety disorders impaired the health-related quality of life, and decrease BS benefits, suggesting that BS does not result in consistent long-term reductions in mental disorders. ${ }^{83}$ Programs including psychological followup after BS are often not available, therefore very little is known about the best approach to improve mental health after BS. ${ }^{84}$ However, once a psychiatric disease has been properly framed, these patients should be referred to a psychiatric and management through both psychotherapy and medication should be planned.

\section{Nutritional Assessment}

Sarcopenia, defined as a progressive and generalized loss of skeletal muscle mass, strength, and function ${ }^{85}$ as well as frailty ${ }^{86}$ have been recently identified as detrimental comorbidities in patients with cirrhosis. In the evaluation of the BS candidate with liver disease, a preoperative assessment of sarcopenia may be recommended. Indeed, the gold standard for the quantification of skeletal muscle mass in liver disease is nowadays considered skeletal muscle index (SMI). This is obtained normalizing the cross-sectional area of the psoas muscle (additionally para-spinal and abdominal wall muscles) acquired with computed tomographic (CT) image analysis at the third lumbar (L3) vertebra. ${ }^{87}$ Low SMI correlates with poorer outcomes in several studies in patients with liver disease. ${ }^{88}$ However, although CT scans are 
frequently performed in cirrhosis, this cannot be considered a point-of-care test for sarcopenia. Therefore ,other reliable and easily available tools, aiming to evaluate performance status, and which indirectly estimate sarcopenia, have been developed. Among them, the Liver Frailty Index is reliable and reproducible ${ }^{89}$ and correlates with prognosis both in cross-sectional studies and in the longitudinal assessment. ${ }^{90}$

To enhance postoperative recovery, protocols aimed at improving physical fitness, such as the enhanced recovery after surgery (ERAS) have been applied also in the BS setting. They include physical pre-habilitation and rehabilitation programs. A meta-analysis has shown that despite ERAS protocol reduced the length of stay after BS, pre- and rehabilitation had low or no influence on morbidity. ${ }^{91}$ There is basically no data on the effect of physical activity on counteracting sarcopenia in patients with liver disease and concomitant BS. However, in a recent systematic review on the impact of exercise on physical frailty in patients with chronic liver disease and after-LT, the authors found that exercise can improve physical frailty, particularly functional/aerobic capacity and sarcopenia. ${ }^{92}$
Excessive weight loss and malnutrition play an essential role in liver decompensation after BS. Moreover, obesity is known to be paradoxically associated to some extent of malnutrition, mainly deficiency of vitamin $D$ and iron, which are frequently reported before $\mathrm{BS}^{93}$ and can be enhanced thereafter. Therefore, pre and mainly post-BS assessments of malnutrition are paramount, to counteract multisystemic complications, including liver dysfunction. Protein malnutrition plays a pivotal role in liver disease progression and complications and should be as contrasted as possible in the post-BS scenario. The European Practice Guidelines on nutrition in chronic liver disease suggests that the optimal daily protein intake should not be lower than the recommended 1.2 to $1.5 \mathrm{~g} / \mathrm{kg}^{7}$ Oral multivitamins containing vitamins and trace elements are administered systematically during the weight loss phase (regardless of the intervention) and continuously after BS. Particular attention should be paid to vitamin B12, folic acid, and thiamine to prevent neuropathy and encephalopathy post-BS. Vitamins A, E, and $\mathrm{K}$ as well as zinc, iron, and copper often require to be supplemented. In addition, vitamin $\mathrm{D}$ and calcium deficiency if not properly

Table 1 Dietary recommendations and supplementation to avoid deficiency and malnutrition after BS adapted for patient with liver disease $e^{7,77,94}$

\begin{tabular}{|c|c|}
\hline & Recommendations \\
\hline Protein intake & $\begin{array}{l}\text { - Should constitute } 10 \text { to } 35 \% \text { of daily caloric intake. } \\
\text { - In patient with liver disease is required a protein intake between } 1.2 \text { and } 1.5 \mathrm{~g} / \mathrm{kg} \text { while during the active weight loss } \\
\text { phase BPD may require } 1.5 \text { to } 2.0 \mathrm{~g} / \mathrm{kg} \text { body weight per day. }\end{array}$ \\
\hline Carbohydrates & $\begin{array}{l}\text { - Early postoperative- }-50 \mathrm{~g} / \mathrm{d} \text {. } \\
\text { - As diet intake increases }-130 \mathrm{~g} / \mathrm{d} \text {. }\end{array}$ \\
\hline Fat & - $20-35 \%$ of the daily caloric intake; bulk of the fat intake should be unsaturated fat. \\
\hline $\begin{array}{l}\text { Vitamin D } \\
\text { and calcium }\end{array}$ & $\begin{array}{l}\text { - Recommended preventative dose of vitamin D and calcium should be based on serum vitamin D levels, calcium and PTH. } \\
\text { - Recommended vitamin D3 dose is 3,000 IU daily. } \\
\text { - Calcium citrate preferentially in } 2 \text { doses, especially if with PPI. }\end{array}$ \\
\hline Iron & $\begin{array}{l}\text { - Males and patients without a history of anemia: iron from multivitamin supplementation. } \\
\text { - Menstruating females and patients with RYGB, SG, or BPD: 45-60 mg of elemental iron daily. } \\
\text { - Oral supplementation separately from calcium and PPI. }\end{array}$ \\
\hline Folate & $\begin{array}{l}\text { - Regular folate daily dose from multivitamin supplementation. } \\
\text { - } 800-1,000 \mu \text { g oral folate daily in women of child-bearing age. }\end{array}$ \\
\hline Thiamine & $\begin{array}{l}\text { - } 50-100 \mathrm{mg} \text { daily dose of thiamine from a B-complex supplement or high-potency multivitamin. } \\
\text { - When a thiamine deficiency is suspected it should be treated before or in the absence of laboratory confirmation and } \\
\text { monitored/evaluated for resolution of signs and symptoms due to potentially severe/unavertable condition. In this } \\
\text { setting parenteral administration should be preferred and magnesium sulfate added as co-factor. } \\
\text { - In patients with suspected AUD the treatment is started early. }\end{array}$ \\
\hline Vitamin B12 & $\begin{array}{l}\text { - Regular B12 daily dose from multivitamin or B-complex supplementation. } \\
\text { - Supplementary dose should be evaluated according to B12 level and monthly parenteral administration should be } \\
\text { considered }(1,000 \mu \mathrm{g}) \text {. }\end{array}$ \\
\hline Zinc and copper & $\begin{array}{l}\text { - } 4 \text { RDA of zinc and copper as part of routine multivitamin and mineral supplementation. } \\
\text { - Ratio of } 8-15 \mathrm{mg} \text { of supplemental zinc per } 1 \mathrm{mg} \text { of copper to minimize the risk of copper deficiency. }\end{array}$ \\
\hline $\begin{array}{l}\text { Vitamin } \\
\mathrm{A}, \mathrm{E} \text { and } \mathrm{K}\end{array}$ & $\begin{array}{l}\text { - Dosage as part of routine multivitamin and mineral supplementation. } \\
\text { - Higher maintenance doses of fat-soluble vitamins may be required for BS-patients with a previous history of deficiency, } \\
\text { - Mregnancy, or cholestasis present (evaluate parenteral administration). } \\
\text { supplem plasma vitamins concentration, retinol-binding protein and prothrombin time can help in monitoring }\end{array}$ \\
\hline $\begin{array}{l}\text { Behavioral } \\
\text { measurements }\end{array}$ & $\begin{array}{l}\text { Aim to avoid discomfort while the patients eat after BS } \\
\text { - Eat slowly and chew food extensively. } \\
\text { - Little meals, eventually increase the frequency. } \\
\text { - Eat no more than satiety. } \\
\text { - Avoid taking food and beverages at the same time. }\end{array}$ \\
\hline
\end{tabular}

Abbreviations: AUD, alcohol use disorder; BPD biliopancreatic diversion; BS, bariatric surgery; PPI, proton pump inhibitor; PTH, parathyroid hormone; RDA, recommended dietary allowance; RYGB, Roux-en-Y-gastric-bypass; SG, sleeve gastrectomy. 


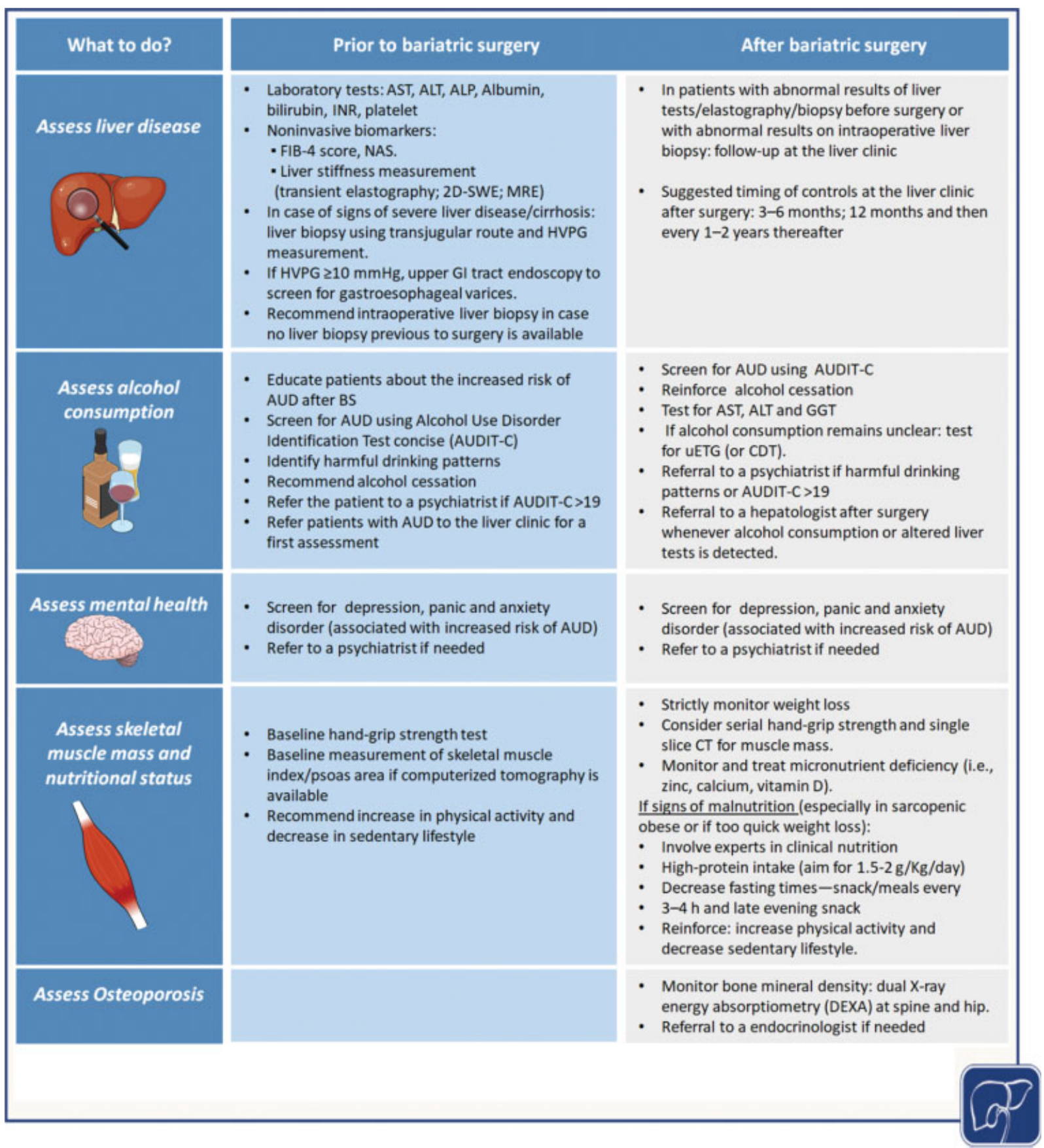

Fig. 4 Proposed actions to identify and follow-up liver disease and to potentially avoid severe decompensation in patients undergoing BS. Abbreviations: BS, bariatric surgery; NAS, NAFLD Fibrosis Score; FIB-4, fibrosis-4 index; HVPG, hepatic venous pressure gradient; AUD, alcohol use disorder; 2D-SWE, two dimensional share wave elastography; MRE, magnetic resonance elastography; AUDIT, alcohol use disorders identification test; $C D T$, serum carbohydrate-deficient transferrin; $\mathrm{UETG}$, with urinary ethyl glucuronide; $\mathrm{CT}$, computed tomography.

counteract can worsen bone loss, which is also affected by the weight loss per se. ${ }^{94}$ Bone disease assessment requires bone density scan, adequate therapy, and correct followup. - Table 1 describes the dietary recommendations and supplementation to avoid deficiency and malnutrition after BS. 7,77,94

Patients who present with liver decompensation after BS should receive a nutritional assessment by nutrition specialists. They should receive oral supplementation targeting 35 to $40 \mathrm{kcal} / \mathrm{kg} / \mathrm{day}$, including the recommended protein intake of 1.2 to $1.5 \mathrm{~g} / \mathrm{kg} / \mathrm{day}^{7}$ by high-protein liquid supplement, plus the substitution of vitamins and trace elements. Patients not achieving sufficient oral caloric intake may require enteral nutrition supplemented through nasojejunal tube (NJT); if patients show intolerance to $\mathrm{NJT}$, they might require temporary parenteral nutrition or undergo bariatric re-intervention. Laparoscopic reversal of BS has been described as improving malnutrition $^{95}$ and consequently clinical stabilization of liver disease. $^{20}$ 


\section{Alternatives to BS in Patients with Cirrhosis}

In the last decade, bariatric endoscopy procedures have emerged as a minimally invasive alternative to surgery. ${ }^{96}$ Endoscopic therapies include duodenal and gastric devices, such as intragastric balloons. ${ }^{97,98}$ Safety of these procedures is likely higher than that of surgery, but data in patients with advanced chronic liver disease/cirrhosis is lacking, and more research is needed to clarify the real risk and benefits in these patients. ${ }^{99}$

\section{Conclusion}

BS can be an appropriate and effective treatment strategy for patients with severely obese patients due to NAFLD or other chronic liver diseases, including selected patients with compensated cirrhosis. However, a subset of patients may show a progression of liver disease to a cirrhosis stage, and can suffer from short or long-term liver dysfunction and complications of portal hypertension, requiring even LT after BS. New findings seem to highlight that severe malnutrition related to excessive weight loss after BS and de novo alcohol misuse might have a role in the deterioration of liver function after BS. There are possible pathophysiological mechanisms related to these factors. Therefore, strict follow-up of weight loss with adequate supplementation of vitamins and trace elements is the measure to avoid malnutrition and sarcopenia. Prevention and early recognition of alcohol misuse pre-and post-surgery is a major unmet need. Patients should be educated about possible alcohol metabolism changes that may make them more susceptible to new or worsening alcohol misuse after BS. An overview of the approach to apply before and after-BS to potentially avoid liver-related complications is proposed in -Fig. 4. A multidisciplinary approach is the mainstay of these patients, and future research is needed on risk factors and the impact of these interventions to prevent liver-related complications.

\section{Main Concepts and Learning Points}

- Bariatric surgery (BS) can lead to improvement of liver disease in patients with nonalcoholic steatohepatitis.

- BS can be considered in selected patients with compensated cirrhosis without severe portal hypertension.

- A careful assessment of portal hypertension including hepatic venous pressure gradient measurement and endoscopy is indicated in patients with signs of cirrhosis before BS.

- Approximately $10 \%$ of BS patients with liver disease can experience progression of liver disease and develop severe decompensation or liver failure requiring liver transplantation.

- Severe malnutrition linked to excessive weight loss, and alcohol misuse are major risk factors for decompensation of liver disease after BS.

- Alcohol abstinence and nutritional interventions can allow for stabilization and recompensation of liver disease.
- Multidisciplinary teams able to early detect malnutrition, alcohol consumption, and psychiatric disorders after BS are an unmet need in this field.

Authors' Contributions

Y.P.M. and C.B. contributed towards conception, literature review, and drafting the manuscript; K.D.W. contributed towards critical revision of the manuscript for important intellectual content and editing the manuscript; A.B. contributed towards conception, critical revision of the manuscript for important intellectual content, drafting, and editing the manuscript. All the authors approved the final version of the manuscript.

Funding

Y.P.M. and C.B. received financial support from the Stiftung für Leberkrankheiten Bern.

Conflicts of Interest

None declared.

\section{References}

1 NCD Risk Factor Collaboration (NCD-RisC) Worldwide trends in body-mass index, underweight, overweight, and obesity from 1975 to 2016: a pooled analysis of 2416 population-based measurement studies in 128.9 million children, adolescents, and adults. Lancet 2017;390(10113):2627-2642

2 English WJ, DeMaria EJ, Hutter MM, et al. American Society for Metabolic and Bariatric Surgery 2018 estimate of metabolic and bariatric procedures performed in the United States. Surg Obes Relat Dis 2020;16(04):457-463

3 Meek CL, Lewis HB, Reimann F, Gribble FM, Park AJ. The effect of bariatric surgery on gastrointestinal and pancreatic peptide hormones. Peptides 2016;77:28-37

4 Ryan KK, Tremaroli V, Clemmensen C, et al. FXR is a molecular target for the effects of vertical sleeve gastrectomy. Nature 2014; 509(7499):183-188

5 Adams TD, Davidson LE, Hunt SC. Weight and metabolic outcomes 12 years after gastric bypass. N Engl J Med 2018;378(01):93-96

6 Thereaux J, Lesuffleur T, Czernichow S, et al. Long-term adverse events after sleeve gastrectomy or gastric bypass: a 7-year nationwide, observational, population-based, cohort study. Lancet Diabetes Endocrinol 2019;7(10):786-795

7 European Association for the Study of the Liver. Electronic address: easloffice@easloffice.eu European Association for the Study of the Liver. EASL Clinical Practice Guidelines on nutrition in chronic liver disease. J Hepatol 2019;70(01):172-193

8 Belle SH, Berk PD, Chapman WH, et al; LABS Consortium. Baseline characteristics of participants in the Longitudinal Assessment of Bariatric Surgery-2 (LABS-2) study. Surg Obes Relat Dis 2013;9 (06):926-935

9 Eslam M, Newsome PN, Sarin SK, et al. A new definition for metabolic dysfunction-associated fatty liver disease: an international expert consensus statement. J Hepatol 2020

10 Chalasani N, Younossi Z, Lavine JE, et al. The diagnosis and management of nonalcoholic fatty liver disease: practice guidance from the American Association for the Study of Liver Diseases. Hepatology 2018;67(01):328-357

11 Froylich D, Corcelles R, Daigle C, Boules M, Brethauer S, Schauer P. Effect of Roux-en-Y gastric bypass and sleeve gastrectomy on nonalcoholic fatty liver disease: a comparative study. Surg Obes Relat Dis 2016;12(01):127-131

12 von Schönfels W, Beckmann JH, Ahrens M, et al. Histologic improvement of NAFLD in patients with obesity after bariatric 
surgery based on standardized NAS (NAFLD activity score). Surg Obes Relat Dis 2018;14(10):1607-1616

13 Fakhry TK, Mhaskar R, Schwitalla T, Muradova E, Gonzalvo JP, Murr MM. Bariatric surgery improves nonalcoholic fatty liver disease: a contemporary systematic review and meta-analysis. Surg Obes Relat Dis 2019;15(03):502-511

14 Lassailly G, Caiazzo R, Buob D, et al. Bariatric surgery reduces features of nonalcoholic steatohepatitis in morbidly obese patients. Gastroenterology 2015;149(02):379-388, quiz e15-e16

15 Lee Y, Doumouras AG, Yu J, et al. Complete resolution of nonalcoholic fatty liver disease after bariatric surgery: a systematic review and meta-analysis. Clin Gastroenterol Hepatol 2019;17 (06):1040-1060.e11

16 Chavez-Tapia NC, Tellez-Avila FI, Barrientos-Gutierrez T, MendezSanchez N, Lizardi-Cervera J, Uribe M. Bariatric surgery for nonalcoholic steatohepatitis in obese patients. Cochrane Database Syst Rev 2010;(01):CD007340

17 Eilenberg M, Langer FB, Beer A, Trauner M, Prager G, Staufer K. Significant liver-related morbidity after bariatric surgery and its reversal-a case series. Obes Surg 2018;28(03):812-819

18 Mahawar KK, Parmar C, Graham Y, et al. Monitoring of liver function tests after Roux-en-Y gastric bypass: an examination of evidence base. Obes Surg 2016;26(10):2516-2522

19 Addeo P, Cesaretti M, Anty R, Iannelli A. Liver transplantation for bariatric surgery-related liver failure: a systematic review of a rare condition. Surg Obes Relat Dis 2019;15(08):1394-1401

20 Mendoza YP, Becchetti C, Wan T, et al. Malnutrition and alcohol in patients presenting with severe complications of cirrhosis after laparoscopic bariatric surgery. Obes Surg 2021;31(06): 2817-2822

21 Pestana L, Swain J, Dierkhising R, Kendrick ML, Kamath PS, Watt KD. Bariatric surgery in patients with cirrhosis with and without portal hypertension: a single-center experience. Mayo Clin Proc 2015;90(02):209-215

22 Patton H, Heimbach J, McCullough A. AGA clinical practice update on bariatric surgery in cirrhosis: expert review. Clin Gastroenterol Hepatol 2021;19(03):436-445

23 Mosko JD, Nguyen GC. Increased perioperative mortality following bariatric surgery among patients with cirrhosis. Clin Gastroenterol Hepatol 2011;9(10):897-901

24 Are VS, Knapp SM, Banerjee A, et al. Improving outcomes of bariatric surgery in patients with cirrhosis in the United States: a nationwide assessment. Am J Gastroenterol 2020;115(11): 1849-1856

25 Jan A, Narwaria M, Mahawar KK. A systematic review of bariatric surgery in patients with liver cirrhosis. Obes Surg 2015;25(08): 1518-1526

26 Izzy M, Angirekula M, Abu Dayyeh BK, Bazerbachi F, Watt KD. Bariatric surgery proves long-term benefit in patients with cirrhosis. Gastroenterol Rep (Oxf) 2020;1-5

27 Reverter E, Cirera I, Albillos A, et al. The prognostic role of hepatic venous pressure gradient in cirrhotic patients undergoing elective extrahepatic surgery. J Hepatol 2019;71(05):942-950

28 Hanipah ZN, Punchai S, McCullough A, et al. Bariatric surgery in patients with cirrhosis and portal hypertension. Obes Surg 2018; 28(11):3431-3438

29 Bosch J, Abraldes JG, Berzigotti A, García-Pagan JC. The clinical use of HVPG measurements in chronic liver disease. Nat Rev Gastroenterol Hepatol 2009;6(10):573-582

30 Markowitz JS, Seu P, Goss JA, et al. Liver transplantation for decompensated cirrhosis after jejunoileal bypass: a strategy for management. Transplantation 1998;65(04):570-572

31 Lowell JA, Shenoy S, Ghalib R, et al. Liver transplantation after jejunoileal bypass for morbid obesity. J Am Coll Surg 1997;185 (02):123-127

32 Geerts A, Darius T, Chapelle T, et al. The multicenter Belgian survey on liver transplantation for hepatocellular failure after bariatric surgery. Transplant Proc 2010;42(10):4395-4398
33 Ayloo S, Buchs NC, Addeo P, Bianco FM, Giulianotti PC. Robotassisted sleeve gastrectomy for super-morbidly obese patients. J Laparoendosc Adv Surg Tech A 2011;21(04): 295-299

34 Lefere S, Stroobant L, Verhelst X, et al. Bariatric surgery patients are at risk for alcoholic liver disease with need for liver transplantation. Obes Surg 2020;30(11):4659-4664

35 Kalinowski P, Paluszkiewicz R, Ziarkiewicz-Wróblewska B, et al. Liver function in patients with nonalcoholic fatty liver disease randomized to Roux-en-Y gastric bypass versus sleeve gastrectomy: a secondary analysis of a randomized clinical trial. Ann Surg 2017;266(05):738-745

36 Adam R, Karam V, Cailliez V, et al; all the other 126 contributing centers (www.eltr.org) and the European Liver and Intestine Transplant Association (ELITA) 2018 annual report of the European Liver Transplant Registry (ELTR)-50-year evolution of liver transplantation. Transpl Int 2018;31(12): 1293-1317

37 Doycheva I, Issa D, Watt KD, Lopez R, Rifai G, Alkhouri N. Nonalcoholic steatohepatitis is the most rapidly increasing indication for liver transplantation in young adults in the United States. J Clin Gastroenterol 2018;52(04):339-346

38 Idriss R, Hasse J, Wu T, et al. Impact of prior bariatric surgery on perioperative liver transplant outcomes. Liver Transpl 2019;25 (02):217-227

39 Sharpton SR, Terrault NA, Posselt AM. Outcomes of sleeve gastrectomy in obese liver transplant candidates. Liver Transpl 2019; 25(04):538-544

40 Heimbach JK, Watt KD, Poterucha JJ, et al. Combined liver transplantation and gastric sleeve resection for patients with medically complicated obesity and end-stage liver disease. Am J Transplant 2013;13(02):363-368

41 Zamora-Valdes D, Watt KD, Kellogg TA, et al. Long-term outcomes of patients undergoing simultaneous liver transplantation and sleeve gastrectomy. Hepatology 2018;68(02):485-495

42 García-Sesma A, Calvo J, Manrique A, et al. Morbidly obese patients awaiting liver transplantation-sleeve gastrectomy: safety and efficacy from a liver transplant unit experience. Transplant Proc 2019;51(01):33-37

43 Lin MY, Tavakol MM, Sarin A, et al. Safety and feasibility of sleeve gastrectomy in morbidly obese patients following liver transplantation. Surg Endosc 2013;27(01):81-85

44 Cheng YL, Elli EF. Outcomes of bariatric surgery after solid organ transplantation. Obes Surg 2020;30(12):4899-4904

45 Lopez-Lopez V, Ruiz-Manzanera JJ, Eshmuminov D, et al. Are we ready for bariatric surgery in a liver transplant program? A metaanalysis. Obes Surg 2021;31(03):1214-1222

46 Suraweera D, Dutson E, Saab S. Liver transplantation and bariatric surgery: best approach. Clin Liver Dis 2017;21(02):215-230

47 Peters RL, Gay T, Reynolds TB. Post-jejunoileal-bypass hepatic disease. Its similarity to alcoholic hepatic disease. Am J Clin Pathol 1975;63(03):318-331

48 Craig RM, Neumann T, Jeejeebhoy KN, Yokoo H. Severe hepatocellular reaction resembling alcoholic hepatitis with cirrhosis after massive small bowel resection and prolonged total parenteral nutrition. Gastroenterology 1980;79(01):131-137

49 Vespasiani-Gentilucci U, Vorini F, Carotti S, et al. Hepatic complications of bariatric surgery : the reverse side of the coin. Acta Gastroenterol Belg 2017;80(04):505-513

50 Mocanu ICS, Costa P, Palma R. Liver failure after bariatric surgeryclinical case and literature review. J Gastroenterol Metabol. 2018; $1: 104$

51 Schrager MA, Metter EJ, Simonsick E, Ble A, Bandinelli S, Lauretani $\mathrm{F}$, et al. Sarcopenic obesity and inflammation in the InCHIANTI study. J Appl Physiol (1985) 2007;102(03):919-925

52 Koo BK, Kim D, Joo SK, et al. Sarcopenia is an independent risk factor for non-alcoholic steatohepatitis and significant fibrosis. J Hepatol 2017;66(01):123-131 
53 Bhanji RA, Narayanan P, Allen AM, Malhi H, Watt KD. Sarcopenia in hiding: The risk and consequence of underestimating muscle dysfunction in nonalcoholic steatohepatitis. Hepatology 2017;66 (06):2055-2065

54 King WC, Chen JY, Courcoulas AP, et al. Alcohol and other substance use after bariatric surgery: prospective evidence from a U.S. multicenter cohort study. Surg Obes Relat Dis 2017; 13(08):1392-1402

55 Orellana ER, Jamis C, Horvath N, Hajnal A. Effect of vertical sleeve gastrectomy on alcohol consumption and preferences in dietary obese rats and mice: a plausible role for altered ghrelin signaling. Brain Res Bull 2018;138:26-36

56 Mellinger JL, Shedden K, Winder GS, et al. Bariatric surgery and the risk of alcohol-related cirrhosis and alcohol misuse. Liver Int 2021;41(05):1012-1019

57 Ibrahim N, Alameddine M, Brennan J, Sessine M, Holliday C, Ghaferi AA. New onset alcohol use disorder following bariatric surgery. Surg Endosc 2019;33(08):2521-2530

58 King WC, Chen JY, Mitchell JE, et al. Prevalence of alcohol use disorders before and after bariatric surgery. JAMA 2012;307(23): 2516-2525

59 Svensson PA, Anveden Å, Romeo S, et al. Alcohol consumption and alcohol problems after bariatric surgery in the Swedish obese subjects study. Obesity (Silver Spring) 2013;21(12): 2444-2451

60 Steffen KJ, Engel SG, Wonderlich JA, Pollert GA, Sondag C. Alcohol and other addictive disorders following bariatric surgery: prevalence, risk factors and possible etiologies. Eur Eat Disord Rev 2015;23(06):442-450

61 Orellana ER, Covasa M, Hajnal A. Neuro-hormonal mechanisms underlying changes in reward related behaviors following weight loss surgery: ootential pharmacological targets. Biochem Pharmacol 2019;164:106-114

62 Johnson PM, Kenny PJ. Dopamine D2 receptors in addiction-like reward dysfunction and compulsive eating in obese rats. Nat Neurosci 2010;13(05):635-641

63 Steele KE, Prokopowicz GP, Schweitzer MA, et al. Alterations of central dopamine receptors before and after gastric bypass surgery. Obes Surg 2010;20(03):369-374

64 Reddy IA, Wasserman DH, Ayala JE, Hasty AH, Abumrad NN, Galli A. Striatal dopamine homeostasis is altered in mice following Roux-en-Y gastric bypass surgery. ACS Chem Neurosci 2014;5 (10):943-951

65 Blackburn AN, Hajnal A, Leggio L. The gut in the brain: the effects of bariatric surgery on alcohol consumption. Addict Biol 2017;22 (06):1540-1553

66 Acevedo MB, Teran-Garcia M, Bucholz KK, et al. Alcohol sensitivity in women after undergoing bariatric surgery: a cross-sectional study. Surg Obes Relat Dis 2020;16(04):536-544

67 Ivezaj V, Benoit SC, Davis J, et al. Changes in alcohol use after metabolic and bariatric surgery: predictors and mechanisms. Curr Psychiatry Rep 2019;21(09):85

68 European Association for Study of Liver Asociacion Latinoamericana para el Estudio del Higado. EASL-ALEH clinical practice guidelines: non-invasive tests for evaluation of liver disease severity and prognosis. J Hepatol 2015;63(01):237-264

69 Newsome PN, Cramb R, Davison SM, et al. Guidelines on the management of abnormal liver blood tests. Gut 2018;67(01): 6-19

70 Castera L, Friedrich-Rust M, Loomba R. Noninvasive assessment of liver disease in patients with nonalcoholic fatty liver disease. Gastroenterology 2019;156(05):1264-1281.e4

71 Papatheodoridi M, Hiriart JB, Lupsor-Platon M, et al. Refining the Baveno VI elastography criteria for the definition of compensated advanced chronic liver disease. J Hepatol 2020

72 Neuberger J, Patel J, Caldwell H, et al. Guidelines on the use of liver biopsy in clinical practice from the British Society of Gastroen- terology, the Royal College of Radiologists and the Royal College of Pathology. Gut 2020;69(08):1382-1403

73 Mahmud N, Fricker Z, Hubbard RA, et al. Risk prediction models for post-operative mortality in patients with cirrhosis. Hepatology 2021;73(01):204-218

74 Martin Mateos R, Garcia de la Filia Molina I, Albillos A. Pre-surgical risk assessment in patients with cirrhosis. Acta Gastroenterol Belg 2020;83(03):449-453

75 Parikh M, Johnson JM, Ballem NAmerican Society for Metabolic and Bariatric Surgery Clinical Issues Committee. ASMBS position statement on alcohol use before and after bariatric surgery. Surg Obes Relat Dis 2016;12(02):225-230

76 Miller-Matero LR, Coleman JP, LaLonde L, Martens KM, Hamann A, Carlin AM. Patient recall of education about the risks of alcohol use following bariatric surgery. Obes Surg 2019;29(08):2707-2710

77 Mechanick JI, Youdim A, Jones DB, et al; American Association of Clinical Endocrinologists Obesity Society American Society for Metabolic \& Bariatric Surgery. Clinical practice guidelines for the perioperative nutritional, metabolic, and nonsurgical support of the bariatric surgery patient-2013 update: cosponsored by American Association of Clinical Endocrinologists, The Obesity Society, and American Society for Metabolic \& Bariatric Surgery. Obesity (Silver Spring) 2013;21 (Suppl 1):S1-S27

78 El Sherif O, Dhaliwal A, Newsome PN, Armstrong MJ. Sarcopenia in nonalcoholic fatty liver disease: new challenges for clinical practice. Expert Rev Gastroenterol Hepatol 2020;14(03):197-205

79 Hydes T, Gilmore W, Sheron N, Gilmore I. Treating alcohol-related liver disease from a public health perspective. J Hepatol 2019;70 (02):223-236

80 Heinberg LJ, Ashton K, Coughlin J. Alcohol and bariatric surgery: review and suggested recommendations for assessment and management. Surg Obes Relat Dis 2012;8(03):357-363

81 Connor JP, Haber PS, Hall WD. Alcohol use disorders. Lancet 2016; 387(10022):988-998

82 Sogg S, Lauretti J, West-Smith L. Recommendations for the presurgical psychosocial evaluation of bariatric surgery patients. Surg Obes Relat Dis 2016;12(04):731-749

83 Kalarchian MA, King WC, Devlin MJ, et al. Psychiatric disorders and weight change in a prospective study of bariatric surgery patients: a 3-year follow-up. Psychosom Med 2016;78(03): 373-381

84 Jumbe S, Hamlet C, Meyrick J. Psychological aspects of bariatric surgery as a treatment for obesity. Curr Obes Rep 2017;6(01): 71-78

85 Cruz-Jentoft AJ, Bahat G, Bauer J, et al; Writing Group for the European Working Group on Sarcopenia in Older People 2 (EWGSOP2), and the Extended Group for EWGSOP2. Sarcopenia: revised European consensus on definition and diagnosis. Age Ageing 2019;48(01):16-31

86 Lai JC, Covinsky KE, Dodge JL, et al. Development of a novel frailty index to predict mortality in patients with end-stage liver disease. Hepatology 2017;66(02):564-574

87 Carey EJ, Lai JC, Wang CW, et al; Fitness, Life Enhancement, and Exercise in Liver Transplantation Consortium. A multicenter study to define sarcopenia in patients with end-stage liver disease. Liver Transpl 2017;23(05):625-633

88 Ebadi M, Bhanji RA, Tandon P, Mazurak V, Baracos VE, MontanoLoza AJ. Review article: prognostic significance of body composition abnormalities in patients with cirrhosis. Aliment Pharmacol Ther 2020;52(04):600-618

89 Wang CW, Lebsack A, Chau S, Lai JC. The range and reproducibility of the liver frailty index. Liver Transpl 2019;25(06):841-847

90 Lai JC, Dodge JL, Kappus MR, et al; Multi-Center Functional Assessment in Liver Transplantation (FrAILT) Study. Changes in frailty are associated with waitlist mortality in patients with cirrhosis. J Hepatol 2020;73(03):575-581 
91 Małczak P, Pisarska M, Piotr M, Wysocki M, Budzyński A, Pędziwiatr M. Enhanced recovery after bariatric surgery: systematic review and meta-analysis. Obes Surg 2017;27(01):226-235

92 Williams FR, Berzigotti A, Lord JM, Lai JC, Armstrong MJ. Review article: impact of exercise on physical frailty in patients with chronic liver disease. Aliment Pharmacol Ther 2019;50(09):988-1000

93 Frame-Peterson LA, Megill RD, Carobrese S, Schweitzer M. Nutrient deficiencies are common prior to bariatric surgery. Nutr Clin Pract 2017;32(04):463-469

94 Nuzzo A, Czernichow S, Hertig A, et al. Prevention and treatment of nutritional complications after bariatric surgery. Lancet Gastroenterol Hepatol 2021;6(03):238-251

95 Shah K, Gislason H. Roux-en-Y gastric bypass reversal: a novel technique with functional reversal-case series. Obes Surg 2020; 30(04):1589-1595
96 Orlandini B, Gallo C, Boškoski I, Bove V, Costamagna G. Procedures and devices for bariatric and metabolic endoscopy. Ther Adv Gastrointest Endosc 2020;13:2631774520925647

97 Bazerbachi F, Vargas EJ, Rizk M, et al. Intragastric balloon placement induces significant metabolic and histologic improvement in patients with nonalcoholic steatohepatitis. Clin Gastroenterol Hepatol 2021;19(01):146-154.e4

98 Lee YM, Low HC, Lim LG, et al. Intragastric balloon significantly improves nonalcoholic fatty liver disease activity score in obese patients with nonalcoholic steatohepatitis: a pilot study. Gastrointest Endosc 2012;76(04):756-760

99 Salomone F, Sharaiha RZ, Boškoski I. Endoscopic bariatric and metabolic therapies for non-alcoholic fatty liver disease: evidence and perspectives. Liver Int 2020;40(06): $1262-1268$ 